\title{
Informativity of the Vibration Signal of the Spindle Unit of the Metal Cutting Machine
}

\author{
Alexander Denisenko ${ }^{1, *}$ and Viktor Mikhailov ${ }^{1}$ \\ ${ }^{1}$ Samara State Technical University, Samara, Russian Federation
}

\begin{abstract}
Monitoring the condition of the spindle units of modern metalcutting machines by methods of non-selective diagnostics involves the possibility of installing monitoring sensors in places with maximum vibration information. In this regard, the assessment of the informative value of the vibration field of the spindle unit, which can be carried out in advance, taking into account the design features, geometric and dimensional characteristics, is an urgent task. Based on the energy approach, a computational model based on the median planes of the walls is proposed using the example of a universal lathe spindle unit. On the basis of the calculated model, the energy balance equations are drawn up taking into account the conditions for the transmission of vibration power between the walls of the housing. The dependences that allow us to calculate the coefficients that take into account the absorption of vibration energy by the walls of the housing are given. The solution of the energy balance equations made it possible, based on the level of the vibration power flow, to rank the walls of the spindle unit body by information content,. The resulting model of the vibration field can be used to determine the reference values of vibration velocities that are formed from sources in the absence of defects. This will allow for non-selective diagnostics to detect the occurrence of a defect at an early stage, and in the presence of a defect to assess the level of its development.
\end{abstract}

\section{Introduction}

The increase in the productivity of modern metal-cutting equipment is objectively accompanied by an increase in the rotational speeds of the shafts of spindle units (SU). The consequence of this is an increase in the vibro-acoustic activity of the main sources of vibration in the SU: shaft imbalance, belt and gear drives, bearings.

The spindle unit is a complex multi-component system, the technical condition of which is determined by the combined influence of a large number of factors (the type of layout, the geometric dimensions of the elements, their physical and mechanical parameters, etc.). These factors are formed and depend on the quality of manufacturing parts and assembly and change as they are used due to the influence of wear processes.

The transition to the maintenance system of metal-cutting machines according to their actual condition [1] requires the implementation of the possibility of assessing the condition

\footnotetext{
* Corresponding author: sammortor@yandex.ru
} 
of the spindle unit under operating conditions and conditions when the vibration processes characteristic of the spindle unit are significantly intensified, which should be recorded by vibration diagnostics methods [2].

When monitoring the vibration state of complex technical systems, which include metal-cutting machines, usually use a general rule: measurements should be carried out at the point closest to the critical node on rigid structural elements. In this case, the number of conjugate surfaces in the path of propagation of the vibration signal should be minimal [2]. However, since this must be done by methods of non-selective diagnostics and often it is not possible to carry out the control at a point close to the source of vibration disturbance, the problem arises of estimating the level of increase in the vibration signal in the source by analyzing the signal in the form of an elastic wave in the walls of the spindle unit housing available for monitoring. To do this, it is necessary to evaluate the informative value of the vibration signal in the areas of the housing that are available for monitoring. To do this, it is necessary to develop a model that allows you to estimate the level of the vibration signal at the control points, depending on the signal levels in the sources of its occurrence, which determines the state of the node being checked and the development of a possible defect.

The vibration signal travels a considerable distance from the place of its origin to the place of measurement, which leads to its distortion and attenuation. For example, R. B. Randall in [3] points out the general problem of vibration diagnostics, which is that any vibration signal is the sum of the effects from the vibration source and the effects from the path of its transmission.

In the open press, there is a limited number of works devoted to the analysis of the mechanism of transmission of the vibration signal from the place of origin to the place of measurement. These are mainly works in the field of shipbuilding and construction [4...7]. In the field of mechanical engineering, there are separate works by A. N. Chukarin and his employees [8...10], devoted to the formation of the acoustic level of objects that meet the current sanitary standards of working conditions.

\section{Development of the SU vibration field model}

As noted in [11], for complex spatial structures, the description of the vibration field requires the use of multidimensional vectors and matrices, which become less visual and reliable as the systems become more complex. Therefore, a promising approach is to estimate the vibrational power in the system based on the consideration of the vibrational energy flows in the housing objects that are the link with the main sources of vibration (rotating shafts, bearings, gears and belt drives). Therefore, in diffuse vibration fields excited by sources with a wide range of frequency and amplitude characteristics, energy fluxes are the main calculated values [4].

Consider the development of a model of the vibration field on the example of the SU universal lathe mod. 16B16KP (Fig. 1).

The SU body is made of cast iron and has the shape of a rectangular parallelepiped with a partition dividing the internal volume, in which the shaft supports are located. Since the top of the spindle unit is closed with a lid that rests on the vertical walls of the model through a rubber seal, the transmission of vibration power through such a connection is insignificant. In this regard, the SU cap is not considered in the calculation model.

Thus, from the point of view of the propagation of vibrations, the SU machine body is a set of plates vibrationally connected to each other in accordance with the geometry of the body, the vibrations of which form the vibration pattern.

In accordance with [11], the total flow of vibrational energy in a certain element of the system is equal to the algebraic sum of flows through individual sections of element 
$N=\sum_{i} N_{i}$, and for complex systems, some terms may have the opposite sign, which indicates the presence of reverse flows of vibrational energy.

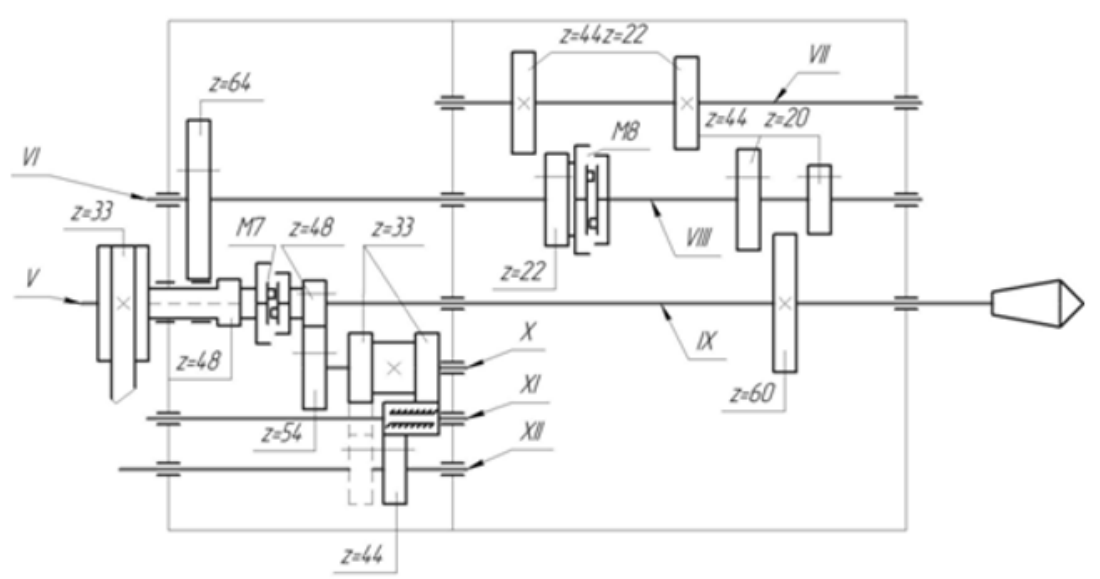

Fig. 1. Kinematic diagram of the spindle unit of the machine mod. 16B16KP

For each such element, an energy balance equation can be written, taking into account the energy input from external sources, the energy exchange between the connected elements, and the energy absorption in the element. The solution of a system of such equations, the number of which is equal to the number of elements that make up the body part, allows you to evaluate the vibration characteristics of the SU, depending on the features of its design.

When developing the model, a number of assumptions have to be made:

- the wall thickness is assumed to be constant;

- bores in the walls are not taken into account;

- energy losses due to sound emission into the surrounding environment are not taken into account;

- energy losses take into account losses in the material of the structure and structural energy losses due to the presence of joints and joints in the structure;

- the vibration signal is transmitted to the housing from internal sources through the bores under the bearings.

The energy approach to solving such problems is widely used in vibroacoustics of engineering structures, such as buildings, aircraft, ships, etc. [4...7].

Despite a number of approximations and assumptions made in these methods, they allow us to obtain relatively simple analytical expressions that agree well with the experiment.

It is most convenient to use energy methods in conditions of diffuse fields characterized by a uniform distribution of energy over the surface of the element. This assumption is very reasonable, given the size of the body parts. With this assumption, the energy of an element in a certain frequency band can be determined by the energy density $w$, and the energy in the entire element in the form of a plate with an area of $S$ is $w S$.

It is assumed that there are several modes of vibration of the element in the frequency band under consideration. It is established [12] that for the existence of a diffuse field in a plate, simultaneous excitation of at least 5 modes of its oscillations is sufficient.

With this in mind, the system of energy balance equations for the stationary process of the $i$-th element can be written in the following form 


$$
N_{i d i s s}+N_{i j}=N_{j i}+N_{i} .
$$

The left part of this equation characterizes the energy lost by the $i$-th element: the first term in the left part of the equation $N_{\text {idiss }}$ describes the energy absorbed by the $i$-th element; the second term-the energy $N_{i j}$ lost by the $i$-th element due to its leakage into other elements; the right part of the equation characterizes the energy entering the $i$-th element: the first term in the right part of the equation describes the incoming energy $N_{j i}$ from the adjacent elements connected to the $i$-th, the second-the energy $N_{i}$ entering the $i$ th element from outside through the bores of the wall.

Based on equation (1), for the case when elements $j, r, t$ and $v$ are associated with element $i$, we can write [8]:

$$
\begin{aligned}
& \delta_{i} S_{i} q_{i}+\left(\alpha_{i-j} l_{i-j}+\alpha_{i-r} l_{i-r}+\alpha_{i-t} l_{i-t}+\alpha_{i-v} l_{i-v}\right) q_{i}= \\
& =\left(\alpha_{j-i} l_{j-i} q_{j}+\alpha_{r-i} l_{r-i} q_{r}+\alpha_{t-i} l_{t-i} q_{t}+\alpha_{v-i} l_{v-i} q_{v}\right)+\sum_{k=1}^{k_{i}} N_{i k},
\end{aligned}
$$

where $\delta_{i}$ is the energy absorption coefficients in the $i$-th wall of the housing, $\mathrm{m}^{-1} ; S_{i}$ is the area of the $i$-th wall, $\mathrm{m}^{2} ; \alpha_{i-\ldots} ; \alpha_{\ldots-i}$ is the vibrational power transfer coefficients between the adjacent walls of the housing (in the index, the sequence corresponds to the direction of the vibrational energy flow, respectively, the first index means the plate from which the power is transmitted, and the second-to which); $l_{i-\ldots}=l_{\ldots-i}$ is the length of the contact line between the two plates, $\mathrm{m} ; q_{i} ; q_{j} ; q_{r} ; q_{t} ; q_{v}$ is the vibrational power flows in the $i$-th , $j$-th, $r$-th, $t$-th and $v$-th wall of the housing, respectively, $\mathrm{W} / \mathrm{m} ; k_{i}$-the number of bearings in the $i$-th wall; $N_{i k}$-vibration power introduced into the housing through the $k$ th bearing assembly in the $i$-th wall of the housing, W.

Thus, the calculation model based on the median planes of the walls will represent a structure consisting of 9 flat rectangular plates, three of which (No. 3, 6 and 7) have vibration sources (Fig. 2). The wall thicknesses are shown in Table 1.

The plates on each of their rectilinear boundaries are connected to one (angular connection) or two plates (t-shaped connection). The latter case takes place in the connection of plates No. 1, 2 and 7 and No. 4, 5 and 7.

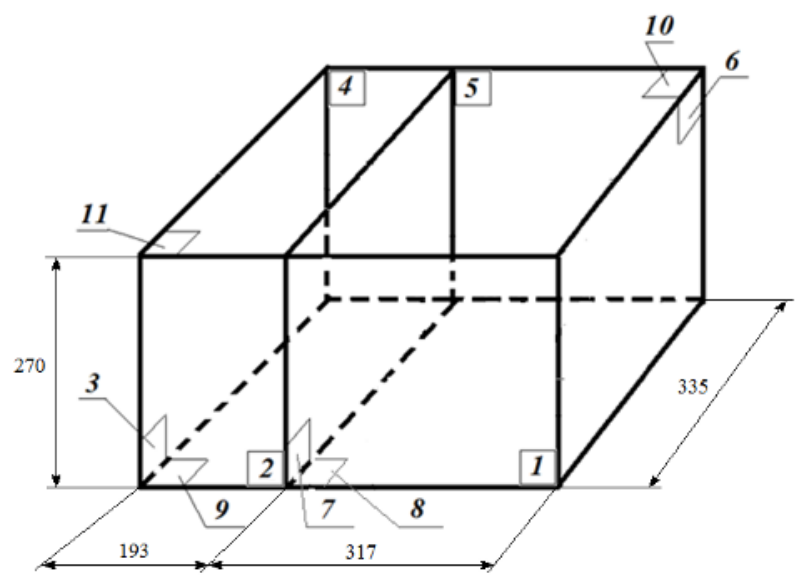

Fig. 2. Calculation model of the SU, built on the median planes of the walls 
Table 1. Wall thickness of the SU machine body 16B16KP

\begin{tabular}{|c|c|c|c|c|c|c|c|c|c|}
\hline Plate (wall) number & 1 & 2 & 3 & 4 & 5 & 6 & 7 & 8 & 9 \\
\hline Plate (wall) thickness $h_{i}, \mathrm{~mm}$ & 40 & 40 & 48 & 45 & 45 & 30 & 30 & 65 & 65 \\
\hline
\end{tabular}

Using the generalized equation (2), we write down the energy balance equations for the accepted calculation model:

$$
\begin{aligned}
& \delta_{1} S_{1} q_{1}+\left(\alpha_{1-2} l_{1-2}+\alpha_{1-6} l_{1-6}+\alpha_{1-7} l_{1-7}+\alpha_{1-8} l_{1-8}\right) q_{1}= \\
& =\alpha_{2-1} l_{2-1} q_{2}+\alpha_{6-1} l_{6-1} q_{6}+\alpha_{7-1} l_{7-1} q_{7}+\alpha_{8-1} l_{8-1} q_{8} \text {; } \\
& \delta_{2} S_{2} q_{2}+\left(\alpha_{2-1} l_{2-1}+\alpha_{2-3} l_{2-3}+\alpha_{2-7} l_{2-7}+\alpha_{2-9} l_{2-9}\right) q_{2}= \\
& =\alpha_{1-2} l_{1-2} q_{1}+\alpha_{3-2} l_{3-2} q_{3}+\alpha_{7-2} l_{7-2} q_{7}+\alpha_{9-2} l_{9-2} q_{9} \text {; } \\
& \delta_{3} S_{3} q_{3}+\left(\alpha_{3-2} l_{3-2}+\alpha_{3-4} l_{3-4}+\alpha_{3-9} l_{3-9}\right) q_{3}=\alpha_{2-3} l_{2-3} q_{2}+\alpha_{4-3} l_{4-3} q_{4}+\alpha_{9-3} l_{9-3} q_{9}+\sum_{k=1}^{k_{3}} N_{3 k} \text {; } \\
& \delta_{4} S_{4} q_{4}+\left(\alpha_{4-5} l_{4-5}+\alpha_{4-3} l_{4-3}+\alpha_{4-7} l_{4-7}+\alpha_{4-9} l_{4-9}\right) q_{4}= \\
& =\alpha_{5-4} l_{5-4} q_{5}+\alpha_{3-4} l_{3-4} q_{3}+\alpha_{7-4} l_{7-4} q_{7}+\alpha_{9-4} l_{9-4} q_{9} \text {; } \\
& \delta_{5} S_{5} q_{5}+\left(\alpha_{5-4} l_{5-4}+\alpha_{5-6} l_{5-6}+\alpha_{5-7} l_{5-7}+\alpha_{5-8} l_{5-8}\right) q_{5}= \\
& =\alpha_{4-5} l_{4-5} q_{4}+\alpha_{6-5} l_{6-5} q_{6}+\alpha_{7-5} l_{7-5} q_{7}+\alpha_{8-5} l_{8-5} q_{8} \text {; } \\
& \delta_{6} S_{6} q_{6}+\left(\alpha_{6-1} l_{6-1}+\alpha_{6-5} l_{6-5}+\alpha_{6-8} l_{6-8}\right) q_{6}=\alpha_{1-6} l_{1-6} q_{1}+\alpha_{5-6} l_{5-6} q_{5}+\alpha_{8-6} l_{8-6} q_{8}+\sum_{k=1}^{k_{6}} N_{6 k} \text {; } \\
& \delta_{7} S_{7} q_{7}+\left(\alpha_{7-1} l_{7-1}+\alpha_{7-2} l_{7-2}+\alpha_{7-4} l_{7-4}+\alpha_{7-5} l_{7-5}+\alpha_{7-8} l_{7-8}+\alpha_{7-9} l_{7-9}\right) q_{7}= \\
& =\alpha_{1-7} l_{1-7} q_{1}+\alpha_{2-7} l_{2-7} q_{2}+\alpha_{4-7} l_{4-7} q_{4}+\alpha_{5-7} l_{5-7} q_{5}+\alpha_{8-7} l_{8-7} q_{8}+\alpha_{9-7} l_{9-7} q_{9}+\sum_{k=1}^{k 7} N_{7 k} \text {; } \\
& \delta_{8} S_{8} q_{8}+\left(\alpha_{8-1} l_{8-1}+\alpha_{8-5} l_{8-5}+\alpha_{8-6} l_{8-6}+\alpha_{8-7} l_{8-7}+\alpha_{8-9} l_{8-9}\right) q_{8}= \\
& =\alpha_{1-8} l_{1-8} q_{1}+\alpha_{5-8} l_{5-8} q_{5}+\alpha_{6-8} l_{6-8} q_{6}+\alpha_{7-8} l_{7-8} q_{7}+\alpha_{9-8} l_{9-8} q_{9} \text {; } \\
& \delta_{9} S_{9} q_{9}+\left(\alpha_{9-2} l_{9-2}+\alpha_{9-3} l_{9-3}+\alpha_{9-4} l_{9-4}++\alpha_{9-7} l_{9-7}+\alpha_{9-8} l_{9-8}\right) q_{9}= \\
& =\alpha_{2-9} l_{2-9} q_{2}+\alpha_{3-9} l_{3-9} q_{3}+\alpha_{4-9} l_{4-9} q_{4}+\alpha_{7-9} l_{7-9} q_{7}+\alpha_{8-9} l_{8-9} q_{8} \text {. }
\end{aligned}
$$

\section{Determination of energy absorption coefficients in the housing walls}

The energy absorption coefficients in the $i$-th wall of the $\delta_{i}, \mathrm{~m}^{-1}$, housing for the cast-iron housing according to the data of [8] are determined by the expression

$$
\delta_{i}=1,5 \cdot 10^{-3} \eta \sqrt{\frac{f_{0 i}}{h_{i}}}
$$

where $\eta$ is the effective coefficient of vibrational energy loss (for cast iron $\eta=10^{-3}$ [9]); $f_{0 i}$ is the natural frequency of wall vibrations, $\mathrm{Hz}$ :

$$
f_{0 i}=\frac{K_{\alpha}}{2 \pi \cdot a_{i}^{2}} \sqrt{\frac{D_{i}}{\rho \cdot h_{i}}}
$$


where $K_{\alpha}$ is the coefficient depending on the method of fixing the plate; $a_{i}$ is the length of the $i$-th plate, $\mathrm{m} ; \rho$ is the density, $\mathrm{kg} / \mathrm{m}^{3} ; D_{i}$ is the cylindrical stiffness of the plate, $\mathrm{Nm}$ :

$$
D_{i}=\frac{E h_{i}^{3}}{12\left(1-\mu^{2}\right)},
$$

where $E$ is the elastic modulus of the plate material, $\mathrm{Pa} ; \mu$ is the Poisson's ratio for the plate material.

The results of the calculation of the energy absorption coefficients $\delta_{i}$ in the walls of the housing are shown in Table 2.

Table 2. Energy absorption coefficients in the walls of the SU housing of the machine 16B16KP

\begin{tabular}{|c|c|c|c|c|c|}
\hline $\begin{array}{c}\text { Wall } \\
\text { number }\end{array}$ & $\begin{array}{c}\text { Fixing the } \\
\text { plate }\end{array}$ & $\begin{array}{c}\text { The formula for } \\
\text { determining } K_{\alpha}\end{array}$ & $Y$ & $K_{\alpha}$ & $\delta$ \\
\hline 1 & On three sides & $22.37 \sqrt{1+0.14 Y^{2}+0.02 Y^{4}}$ & 1.174 & 24.819 & $3.285 \cdot 10^{-4}$ \\
\hline 2 & On three sides & $22.37 \sqrt{1+0.14 Y^{2}+0.02 Y^{4}}$ & 1.399 & 25.998 & $3.948 \cdot 10^{-4}$ \\
\hline 3 & On three sides & $22.37 \sqrt{1+0.14 Y^{2}+0.02 Y^{4}}$ & 1.241 & 25.141 & $3.129 \cdot 10^{-4}$ \\
\hline 4 & On three sides & $22.37 \sqrt{1+0.14 Y^{2}+0.02 Y^{4}}$ & 1.399 & 25.998 & $3.948 \cdot 10^{-4}$ \\
\hline 5 & On three sides & $22.37 \sqrt{1+0.14 Y^{2}+0.02 Y^{4}}$ & 1.174 & 24.819 & $3.286 \cdot 10^{-4}$ \\
\hline 6 & On three sides & $22.37 \sqrt{1+0.14 Y^{2}+0.02 Y^{4}}$ & 1.241 & 25.141 & $3.128 \cdot 10^{-4}$ \\
\hline 7 & On three sides & $22.37 \sqrt{1+0.14 Y^{2}+0.02 Y^{4}}$ & 1.241 & 25.141 & $3.128 \cdot 10^{-4}$ \\
\hline 8 & On the four sides & $22.37 \sqrt{1+0.61 Y^{2}+Y^{4}}$ & 1.057 & 38.290 & $3.861 \cdot 10^{-4}$ \\
\hline 9 & On the four sides & $22.37 \sqrt{1+0.61 Y^{2}+Y^{4}}$ & 1.736 & 77.236 & $5.484 \cdot 10^{-4}$ \\
\hline \multirow{2}{*}{+} & $\begin{array}{l}\mathrm{a}-\text { large side of the } \\
\text { plate } \\
\mathrm{b}-\text { the smaller side of the } \\
\text { plate } \\
\text { Y=a/b }\end{array}$ & & & \\
\cline { 2 - 6 } & a & & & & \\
\cline { 2 - 6 }
\end{tabular}

\section{Determination of the coefficients of transmission of vibration power between adjacent walls of the housing}

When determining the coefficients of transmission of vibration power during the passage of bending vibrations between two adjacent walls of the housing, you can use the dependence:

$$
\alpha_{i-j}=\frac{\tau_{i-j}}{\pi},
$$

where $\tau_{i-j}$ is the coefficient of passage of the incident wave from the $i$-th plate to the $j$-th plate.

In [7], when considering the problem of determining the coefficients of vibrational power transmission through a set of $n$ semi-infinite plates intersecting along a single line, the expression was obtained using numerical methods

$$
\tau_{i-j}=\tau_{0 i-j} \cdot \psi
$$


where $\tau_{0 i-j}$ is the coefficient of passage of a normally incident wave from the $i$-th plate to the $j$-th plate,

and a graph of the function $\psi$ is plotted, taking into account the ratio of plate thicknesses (Fig. 3).

The possibility of applying the results obtained in [7] in engineering practice is confirmed by their wide use in solving specific problems $[8,9,13]$.

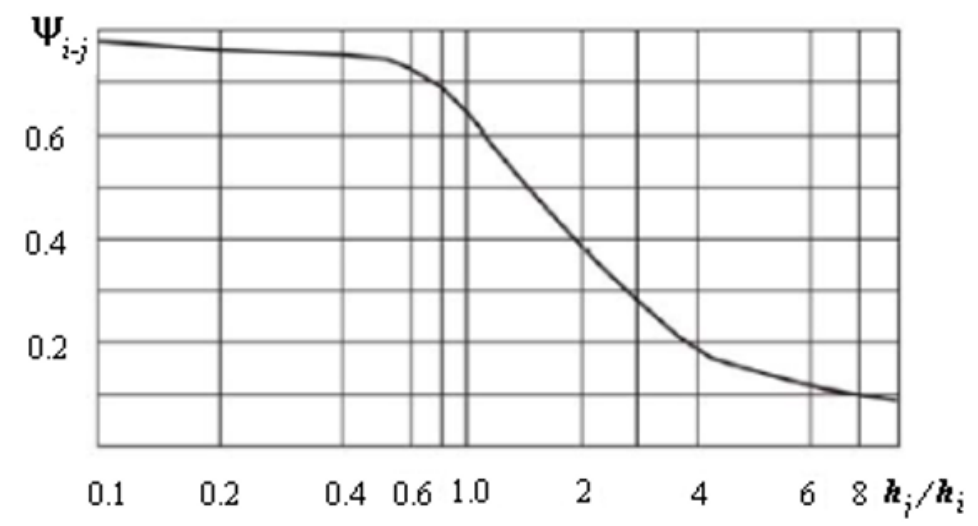

Fig. 3. Dependence of the coefficient $\psi$ on the ratio of plate thicknesses

The coefficient $\tau_{0 i-j}$ significantly depends on the type of connection of the adjacent plates. In [14], the dependences for calculating the coefficient $\tau_{0 i-j}$ of walls connected at an angle of $90^{\circ}$ are given

for the angular joint

$$
\tau_{0 i-j}=\left[\left(\frac{h_{j}}{h_{i}}\right)^{-5 / 4}+\left(\frac{h_{j}}{h_{i}}\right)^{5 / 4}\right]^{-2}
$$

and the T-shaped joint (wall $k$ is a continuation of the wall $i: h_{i}=h_{k}$ )

$$
\tau_{0 i-j}=\left[\sqrt{2} \sigma^{-5 / 4}+\sigma^{5 / 4} / \sqrt{2}\right]^{-2}, \tau_{0 i-k}=\left[2+2 \sigma^{5 / 2}+0,5 \sigma^{5}\right]^{-1},
$$

where $\sigma=\frac{h_{j}}{h_{i}}$.

\section{Approbation}

The solution of the system of equations (3) for the considered computational model in symbolic form is obtained using the Mathcad package:

$$
\begin{aligned}
& q_{1}=4165.261 N_{3}+4172.042 N_{6}+4169.799 N_{7} ; \\
& q_{2}=4154.509 N_{3}+4145.148 N_{6}+4151.947 N_{7} ; \\
& q_{3}=3692.367 N_{3}+3657.987 N_{6}+3662.830 N_{7} ; \\
& q_{4}=3722.529 N_{3}+3712.605 N_{6}+3717.709 N_{7} ; \\
& q_{5}=3710.105 N_{3}+3714.209 N_{6}+3712.252 N_{7} ; \\
& q_{6}=5300.970 N_{3}+5350.126 N_{6}+5304.585 N_{7} ; \\
& q_{7}=5274.299 N_{3}+5271.039 N_{6}+5305.238 N_{7} ;
\end{aligned}
$$




$$
\begin{aligned}
& q_{8}=2783.103 N_{3}+2783.797 N_{6}+2782.947 N_{7} \\
& q_{9}=2810.133 N_{3}+2802.730 N_{6}+2805.311 N_{7} .
\end{aligned}
$$

where $N_{3}, N_{6}, N_{7}$ is the vibration power entering the walls No. 3, 6 and 7, respectively.

The results obtained for $q_{i}$ allow us to rank the walls of the SU housing by information content, based on the level of the vibration power flow: walls No. 6, 7; walls No. 1, 2; walls No. 3, 4, 5; walls No. 8, 9.

The energy fluxes found in the elements of the SU $q_{i}$ housing are related to the oscillation rate of $V_{i}$ of these elements by the dependence [13]

$$
q_{i}=Z_{i} V_{i}^{2}
$$

where $Z_{i}$ is the impedance of the corresponding spindle headstock element, $\mathrm{kg} / \mathrm{s}$.

The dependence (12) allows us to determine the expected values of the wall vibration rates and compare them with the values obtained experimentally.

\section{Conclusions}

The developed model of the SU vibration field allows us to obtain the distribution of vibration power flows along the walls, depending on the conditions of their coupling, the geometry and size of the housing. The obtained dependences of $q_{i}$ on the level of vibration power coming from the main sources make it possible to select the housing locations that are available for monitoring and have the greatest information content. The resulting model of the vibration field can be used to determine the reference values of vibration velocities that are formed from sources in the absence of defects. This will allow for non-selective diagnostics to detect the occurrence of a defect at an early stage, and in the presence of a defect to assess the level of its development.

\section{References}

1. V.I. Pisarev., A.A. Vaganov, A.F. Denisenko, I.O. Tyuterev, Bulletin of the Samara Scientific Center of the Russian Academy of Sciences, 16, 1(2) (2014).

2. Non-destructive testing/ Handbook in 7 volumes, edited by V. V. Klyuev. V 7. Book.2. Vibrodiagnostika, (2005)

3. R.B. Randall, Structural Health Monitoring (Chichester: John Wiles \& Sons. Ltd., 2011)

4. A.S. Nikiforov, S.V. Budrin, Propagation and absorption of sound vibration on ships (Leningrad, Shipbuilding, 1968)

5. S.N. Ovsyannikov, Propagation of sound vibration in civil buildings (Tomsk: Publishing House of the Tomsk State University of Architecture and Civil Engineering, 2000)

6. M. Crocker, A. Price, J. Sound Vib, 9(3) (1969)

7. L.S. Boroditsky, V.M. Spiridonov, Reduction of structural noise in ship premises (Leningrad, Shipbuilding, 1974)

8. V.A. Bondarenko, A.N. Chukarin, Bulletin of the Rostov State University of Railway Transport, 1 (49) (2013) 
9. G.V. Kadubovskaya, A.N. Chukarin, Bulletin of the Don State Technical University, 14, 4 (79) (2014)

10. K.O. Kobzev, A.N. Chukarin, V.A. Bondarenko, Bulletin of the Don State Technical University, 3(86) (2016)

11. Vibrations in engineering: In 6 vols. / Ed. advice: V. N. Chelomey (pred.). Vol. 5. Measurements and tests. - Ed. by M. D. Genkin, (1981)

12. A.S. Nikiforov, Vibration absorption on ships (Leningrad, Shipbuilding, 1979)

13. S.V. Golosnoy, M.V. Ermolov, Online Journal "NAUKOVEDENIE", 9(2) (2017)

14. http://naukovedenie.ru/PDF/28TVN217.pdf (access is free).

15. Handbook of technical Acoustics: Trans. from German./Edited by M. Heckle and H. A. Muller ( Leningrad, Shipbuilding. 1980) 